\title{
Small-on-large fractional derivative-based single-cell model incorporating cytoskeleton prestretch
}

\author{
M. Fraldi, A. Cugno, A.R. Carotenuto, A. Cutolo $\$$ N. M. Pugno, and L. Deseri ${ }^{\ddagger}$
}

\author{
March 8, 2017
}

\begin{abstract}
In the last years, experimental evidences have suggested important direct implications of viscoelasticity of human cells and cell cytoskeleton dynamics on some relevant collective and at single cell behaviors such as migration, adhesion and morphogenesis. As a consequence, the mechanical properties of single cells as well as how cells respond to mechanical stimuli have been -and currently are- at the center of a vivid debate in the scientific community.

By making reference to important experimental findings from the literature which have shown that human metastatic tumor cells are about $70 \%$ softer than benign cells, independently from the cell lines examined, the present authors have very recently theoretically demonstrated that these differences in stiffness might be exploited to mechanically discriminate healthy and cancer cells,
\end{abstract}

\footnotetext{
*fraldi@unina.it(Corresponding author)

Department of Structures for Engineering and Architecture, Polytechnic School, College of Engineering, University of Napoli Federico II - ITALY Interdisciplinary Research Center for Biomaterials, Polytechnic School, College of Engineering, University of Napoli Federico II - ITALY The Institute of Applied Sciences \& Intelligent Systems, National Research Council of Italy - ITALY

†andrea.cugno@unitn.it

Department of Structures for Engineering and Architecture, Polytechnic School, College of Engineering, University of Napoli Federico II - ITALY

Department of Civil, Environmental and Mechanical Engineering, University of Trento - ITALY

$\ddagger$ angelorosario.carotenuto@unina.it

Department of Chemical, Materials and Production Engineering, University of Naples Federico II - ITALY

§arsenio.cutolo@unina.it

Department of Chemical, Materials and Production Engineering, University of Naples Federico II - ITALY

『nicola.pugno@unitn.it

Department of Civil, Environmental and Mechanical Engineering, University of Trento - ITALY

Center of Materials and Microsystems, Bruno Kessler Foundation, Trento, Italy

School of Engineering and Materials Science, Queen Mary University, London, UK

$\|_{\text {deseri@andrew.cmu.edu }}$

Department of Civil, Environmental and Mechanical Engineering, University of Trento - ITALY

Division of Aerospace Eng., Mechanical, Aerospace \& Civil Eng., College of Eng., Design and Physical Sciences. Brunel University London, UK Department of Nanomedicine, Houston Methodist Research Institute - TX-USA
} 
for example through low intensity therapeutic ultrasound. In particular, by means of a generalized viscoelastic paradigm combining classical and fractional derivative-based models, it has been found that selected frequencies (from tens to hundreds $\mathrm{kHz}$ ) are associated to resonance-like phenomena that are prevailing on thermal fluctuations and that could be hence, at least in principle, helpfully utilized for both targeting and selectively attacking tumor cells.

With the aim of investigating the effect of the prestress - for instance induced in protein filaments during cell adhesion- on the overall cell stiffness and, in turn, on its in-frequency response, a simple multiscale scheme is here proposed to bottom-up enrich the spring-pot-based viscoelastic single-cell models, by incorporating finite elasticity and in this way determining, through sensitivity analyses, the role played by the stretched state of the cytoskeletal elements on the cell vibration.

\section{Introduction}

From the mechanical point of view, single human cells can be seen as viscoelastic systems Del Piero and Deseri (1997); Deseri et al. (2006); Fraldi et al. (2015); Haase and Pelling (2015); Tschoegl (1989). However, differently from inorganic materials, living soft matter is inhomogeneous and generally hierarchically organized (Chen and Pugno (2013); Fraldi(2014); Fraldi and Cowin (2004); Huang et al. (2014); Pugno et al. (2012)) and thus reacts -over different time scales- to mechanical stimuli by simultaneously involving protein filaments and supra-molecular and molecular structures present at different scale levels. As a matter of fact, the cell hierarchical organization works as a complex transducer device that converts mechanical signals in biochemical and physical coordinated events which govern the mechanobiology and the mechanosensing of the whole cell, regulating differentiation, growth, morphogenesis, and - through polymerization/depolymerization-based cytoskeleton structural rearrangements - migration and adhesion phenomena at single-cell and in turn at macroscopic (tissue) level as well (Delsanto et al. (2008); DuFort et al. (2011); Guiot et al. (2006); Paszek et al. (2014)).

Three main mechanically relevant structural systems can be recognized in a human cell, a complex factory that makes proteins and tissue materials (Cowin and Doty (2007)): the 10-nm thick, very deformable $(0.1 \sim 1 \mathrm{kPa})$ lipid bilayer (the cell membrane), the gel-like visco-elastic cytosol and the cytoskeleton, the main bearing cell structure constituted by a network of elastic protein filaments 
that are embedded within the cytosol and anchored both to the nucleus and to cell membrane, which mediates mechanical signals, regulates cell shapes during migration and adhesion and somehow protects the cell. Microtubules (tubes with diameters of about 25-nm made up of two subunits of spiraling tubulin), actin and intermediate filaments (actin twisted double- and inter-woven rope-strands of 7-nm and 10-nm in diameter) are the main cytoskeletal filaments whose assembling/disassembling (polymerization/depolymerization) drives cell motility and spreading (Bao and Suresh (2003); Brunner et al.(2009)).

A significant number of scientific works have been dedicated to the analysis of the response of human cells to mechanical stimuli in the last years and, due to the complexity of the systems, several behaviors still remain not completely understood. Recently, it has also experimentally observed that how cells response to ultrasound strongly depends on the associated applied energy and on the related frequencies as well (Schuster et al. (2013)). Cell membrane damaging were furthermore observed in leukemic and in blood cells after ultrasound treatment (Ellwart et al. (1988)), laboratory evidences showing that tumor cells often result more prone to be killed than healthy ones in case of exposure to ultrasound (Lejbkowicz and Salzberg (1997); Lejbkowicz et al. (1993)). Moreover, adequately modulated ultrasounds seem to be additionally capable of decreasing malignant cell growth, inhibiting cell proliferation (Chumakova et al. (2006); Honda et al. (2004)) and stimulating or increasing wound healing (Schuster et al. (2013)), although the authors of these works admit that "the molecular mechanism of ultrasound induced apoptosis has not yet been clearly understood". In this framework, Mizrahi et al. (2012) have recently experimentally observed relevant dynamics involving cytoskeleton remodeling of human airway smooth muscle cells undergoing low intensity ultrasounds administered both at small strains $\left(10^{-5}\right)$ and ultrasonic frequencies $\left(10^{6} \mathrm{~Hz}\right)$ and at moderately large deformation regimes $\left(10^{-1}\right)$ and low (physiological) frequencies $\left(10^{0} \mathrm{~Hz}\right)$.

Although the underlying mechanisms through which cells perceive and transduce mechanical vibrations is still a challenging task, theoretical studies (Fraldi et al. (2015); Or and Kimmel (2009)) have recently explored the possibility that the relative displacement between cell organelles and cytoplasm induced by ultrasonic waves and due to different inertia of the media plays a key role in resonancelike phenomena, suggesting that US-induced mechanical oscillations greater than thermal maximal fluctuations can actually kindle strain regimes at high-frequency and in turn fatigue-like phenomena, in this way altering signaling pathways within the cell and thus inducing multi-molecular complexes 
conformational shift or disrupting at critical frequencies found both around $45 \mathrm{kHz}$ and $1 \mathrm{MHz}$ (Johns (2002)).

The interest on the analysis of the in-frequency response of single-cell systems is further increased by some experimental studies performed in the last years on individual cancer and healthy cells of different types, which have demonstrated that the first ones were about $70 \%$ softer than the latter (Cross et al. (2008, 2007); Faria et al.(2008); Ketene et al. (2012); Lekka et al. (2012a, 1999, 2012b); Li et al. (2008); Nikkhah et al. (2010); Prabhune et al. (2012); Rebelo et al. (2013)), regardless of the cell lines examined and independently from the specific measurement technique used for determining the mechanical properties (Atomic Force Microscopy, Optical tweezers, etc.). These results cuold be in fact helpfully utilized for -at least in principle- mechanically targeting and selectively attacking cancer cells, leading to envisage possible new applications in diagnoses and therapies of cancer diseases (Fraldi et al. (2015); Jonieztz (2012)).

Therefore, motivated by the above mentioned literature findings and with the aim of enriching the modeling of single-cell systems, in the present work it is investigated the effect of the prestress -for instance induced in protein filaments during cell adhesion- on the overall cell stiffness, finally determining its influence on the in-frequency response of the cell. To make this, a simple multiscale scheme incorporating finite elasticity is first proposed to include, by means of a bottom-up homogenization procedure, suitable prestress-modified stiffness values into the viscoelastic single-cell models. Successively, once the analytical expression of the overall elastic stiffness of an adherent cell has been obtained, the identification of some key model parameters (i.e. prestretch and number of "active" filaments) has been determined to fit the realistic stiffness moduli experimentally measured in the literature for several cell types. Finally, after a short presentation of new generalized spring-pot (fractional derivative-based) viscoelastic models, the role played by the stretched state of the cytoskeletal elements on the cell vibration is in detail studied through sensitivity analyses. 


\section{Elemental nonlinear elastic model of an adherent cell}

\section{Influence of prestress and number of cytoskeleton filaments on the single-cell stiffness}

With the aim of deriving the effect of the pre-stretch accumulated in the cytoskeleton filaments on the overall single-cell stiffness, in this section it is presented a simple non-linear elastic model of the cell structure in which the essential features responsible for the mechanical response of the ensemble (e.g., cytoskeleton protein filaments, cell nucleus and interface conditions with a rigid substrate -say the extra-cellular matrix, ECM) are taken into account, in this manner determining the cell elasticity via a bottom-up procedure.

To this purpose, let us consider the sketch in Figure 1. Therein, starting from a generally unknown initial stress-free configuration (Figure 19), the cell is assumed to be in an actual prestretched configuration (say adherent to the ECM, as shown in Figure $1 \mathrm{p}$ ), and then subjected to a small displacement of its nucleus (Figure 1 1 c). In this scheme, as highlighted in the lateral view (see Figure 1), the cell cytoskeleton is modeled through a structure made of symmetrically and radially placed non-linear elastic filaments (or filament strands) anchored to the central nucleus and to the substrate through the focal adhesion points, in this way implicitly assuming that the cell membrane follows the overall geometry of the model. Also, for sake of simplicity, the entire kinematics is projected in the horizontal plane (say the plane defined by the focal adhesion points), so neglecting the minor effects of stress and strain aliquots associated to the out-of-plane filament elongations caused by the cell stretching. In particular, a reference prestretch (denoted by $\lambda_{p}$ ) characterizes the deformed configuration in which the nucleus is constrained by $n$ elastic strings -representing the actin filaments- arranged uniformly around the nucleus and identified by an angle $\phi_{j 0}=j \frac{2 \pi}{n}$.

To find how the cell structure influences the overall cell stiffness when its filaments are prestressed, by making reference to a small-on-large approach, the nucleus is displaced of $u$ in an arbitrary (say horizontal) direction: as a consequence, maintaining prescribed the focal adhesion points, each filament will result to be stressed to follow the nucleus and the corresponding Piola-Kirchhoff stress 
tensor can be generally written as

$$
\mathbf{P}_{j}=\left(\begin{array}{ccc}
P_{L j} & 0 & 0 \\
0 & P_{T j} & 0 \\
0 & 0 & P_{T j}
\end{array}\right)
$$

where -for the -jth filament- $P_{L j}$ denotes the longitudinal stress component and $P_{T j}$ is the transverse stress one, in this case to be set equal to zero. Once the force $f_{u}$ to be applied to the nucleus for obtaining the displacement $u$ is determined, the related equivalent tangent stiffness can be formally derived as follows

$$
K_{e q}=\left.\frac{\partial f_{u}}{\partial u}\right|_{u=0}
$$

Obviously, the force $f_{u}$ - the resultant of the axial forces of the $n$ filaments (see Figure 2) - will depend on $u$, the prestretch $\lambda_{p}$ of each single filament, the initial stiffness (related to the stress-free configuration), the geometrical parameters, the number $n$ of filaments and the constitutive assumption, say the type of hyperelastic law chosen fot the strings. Therefore, one has

$$
f_{u}+\sum_{j=1}^{n} N_{j} \cos \left(\phi_{j}\right)=0
$$

where $N_{j}=P_{L j} A$ is the contribute of the $-j t h$ filament due to the longitudinal stress times the reference cross-section area $A=A_{j}$ and $\phi_{j}$ is the angle of the -jth filament in its current configuration. The constitutive model for the strings is here fixed by following Holzapfel (2000), in the case of compressible Neo-Hookean solids in which the Strain Energy Density Function (SEDF) is written in terms of the first invariant, $I_{1}$, of the right Cauchy-Green tensor $\mathbf{C}=\mathbf{F}^{\mathbf{T}} \mathbf{F}$ (chosen as measure of the deformation) which in the so-called coupled form - where the isochoric and volumetric parts are interacting - is given by:

$$
\Psi_{N H}=\frac{G}{2}\left(I_{1}-3\right)+\frac{G}{2 \beta}\left(J^{-2 \beta}-1\right) \quad \text { with } \quad \beta=\frac{\nu}{1-2 \nu}
$$


where $J=\operatorname{det} \mathbf{F}$ and $G$ and $\nu$ denote the shear modulus and the Poisson's ratio, respectively. The principal stresses will hence depend on the principal stretches in the form

$$
P_{j}=\frac{\partial \Psi_{N H}}{\partial \lambda_{j}}
$$

and furthermore

$$
\begin{aligned}
& P_{L j}=G\left(\lambda_{L j}-\frac{\left(\lambda_{L j} \lambda_{T j}^{2}\right)^{\frac{2 \nu}{2 \nu-1}}}{\lambda_{L j}}\right), \\
& P_{T j}=2 G\left(\lambda_{T j}-\frac{\left(\lambda_{L j} \lambda_{T j}^{2}\right)^{\frac{2 \nu}{2 \nu-1}}}{\lambda_{T j}}\right)
\end{aligned}
$$

Algebraic manipulations lead to observe that prescribing uniaxial stress states in each filament $\left(P_{T j}=\right.$ 0 ) reduces to impose $\lambda_{T}=\lambda_{L}^{-\nu}$, finally obtaining the longitudinal stress as follows

$$
P_{L j}=G \lambda_{L j}\left(1-\lambda_{L j}^{-2(1+\nu)}\right)
$$

The total stretch in the generic $-j t h$ filament strand can be multiplicatively written as:

$$
\lambda_{L j}=\lambda_{p} \lambda_{u j}
$$

in which $\lambda_{p}=\frac{L}{L_{0}}$ is the initial prestretch related to the current filament length $L$ referred to the initial configuration $L_{0}$-whose values are here assumed to be the same for all the elements due to the symmetry of the initial cell shape $-\lambda_{u j}$ being the stretch of the $-j t h$ string due the displacement $u$ and explicitly given by

$$
\lambda_{u j}=\frac{\sqrt{L^{2} \sin ^{2} \phi_{j}+\left(L \cos \phi_{j}-u\right)^{2}}}{L}
$$

where

$$
\sin \phi_{j}=\frac{L \sin \phi_{j 0}}{\sqrt{L^{2} \sin ^{2} \phi_{j 0}+\left(L \cos \phi_{j 0}-u\right)^{2}}}, \cos \phi_{j}=\frac{L \cos \phi_{j 0}-u}{\sqrt{L^{2} \sin ^{2} \phi_{j 0}+\left(L \cos \phi_{j 0}-u\right)^{2}}}
$$


Finally, by substituting eqs (8) and (3) into (2), after some further algebraic manipulations, one obtains the stiffness $K$ which varies with the displacement $u$ as follows

$$
\left.K=-G A \lambda_{p} \sum_{j=1}^{n}\left(\left(1+(1+2 \nu) \lambda_{p}^{-(1+2 \nu)} \lambda_{u j}^{-2(1+\nu)}\right) \cos \phi_{j} \frac{\partial \lambda_{u j}}{\partial u}-\left(\lambda_{u j}-\lambda_{p}^{-2(1+\nu)} \lambda_{u j}^{-(1+2 \nu)}\right) \sin \phi_{j} \frac{\partial \phi_{j}}{\partial u}\right)\right)
$$

from which one finally has

$$
K_{e q}=\left.K\right|_{u=0}=G A L^{-1} \lambda_{p} \sum_{j=1}^{n}\left(1+\lambda_{p}^{-2(1+\nu)}\left(\nu+(1+\nu) \cos 2 \phi_{j 0}\right)\right)
$$

that represents the analytical form -explicitly depending on both the geometrical and mechanical parameters- of the tangent stiffness of the adherent single-cell structure, associated to the imposed displacement $u$. It is worth to notice that, from (13) and for an arbitrary couple of filaments with prescribed angles $\phi_{j 0}$ and $\phi_{j 0}+\pi$ respectively, the prestretch influences the stiffness in a non linear way, whose form depends on the Poisson'ratio $\nu$. It is then natural to ask if the stiffness is monotonic with the prestretch. By calculating the derivative of the $j$-th addend (and its coaxial) in $K_{e q}$, say $K_{e q j}$, with respect to $\lambda_{p}$ and equating it to zero, one finds

$$
\frac{\partial K_{e q j}}{\partial \lambda_{p}}=G A L^{-1} \cos \phi_{j 0} \lambda_{p}\left(1+(1+2 \nu) \lambda_{p}^{-2(1+\nu)}\right)=0
$$

whose in closed-form solution is

$$
\lambda_{p}=|1+2 \nu|^{\frac{1}{1+\nu}}
$$

which gives compatible (positive) stretches for any angle $\phi_{j 0}$ and Poisson ratios belonging to the classical thermodynamically consistent range $]-1, \frac{1}{2}$ [.

From the biomechanical point of view, this enough counter-intuitive result implies that, as the stiffness varies with increasing prestretches, a minimum must be found (see Figure 3 ) and thus -at least in principle- during a monotonic stretching of the substrate or in searching optimal cytoskeleton configuration, an adherent cell could find minimal energy positions at nonzero strains as well. 


\section{Identification of the model parameters to describe actual cell stiffness}

The above nonlinear elastic model has been introduced to quantitatively estimate the effect of prestress and number of filaments on the overall stiffness of an adherent (prestretched) single cell. With reference to the experimentally measured cell stiffness values (see for a synoptic frame the tables in Fraldi et al. (2015)) and by making use of (13), it is possible to determine the equivalent overall elastic Young modulus of the cell in an arbitrary prestretched configuration, $E_{\text {eq }}$, by considering incompressibility condition -that is $G \simeq \frac{E}{3}, G$ being the first Lamé modulus- as follows (the symbol $C_{0 G}=6 \pi G R$ will be afterwards used for the stiffness)

$$
E_{e q} \simeq \frac{3 K_{e q}}{6 \pi R}
$$

where $R$ represents the cell nucleus radius, as reported by Or and Kimmel (2009). This expression which will be used in the following viscoelastic schemes- implicitly takes into account the prestretch as well as the number of filaments, all these parameters being included in $K_{e q}$. As a consequence, the formula furnishes a direct first estimation of the equivalent cell Young modulus $E_{e q}$-once all the mechanical and geometrical parameters are known- but, because of its elementary structure, it can be also used to identify the number of "active" cytoskeleton elements in an experimental measurement, as well as to determine the average prestress of an adherent cell.

In Figure 4 are illustrated the results of the parametric analyses conducted on the equivalent stiffness for three values of the Poisson's ratio of the filaments $(\nu=0,0.25,0.5)$, initial filament length $L_{0}$ equal to $50 \mu \mathrm{m}$ and circular cross sections with diameters of $7 \mathrm{~nm}$, all these values being coherent with the literature data. In particular, the equivalent cell Young moduli of a cell have been carried out by both considering 75 active protein filaments for a single cell strand, making variable the prestretch (see Figure 4 ), and complementary prescribing a prestretch $\left(\lambda_{p}=1.3\right)$, thus plotting the cell stiffness against the number of filaments (see Figure 4p). Both the graphics show how the whole range of elastic moduli measured through different techniques and reported in the literature for a vast class of cell lines (Fraldi et al. (2015)) can be obtained with a good agreement, modulating the prestretch and the number of "active" filaments within experimentally documented intervals. An instructive numerical example can be easily done by considering the case of cell stiffness measured by Cross et al Cross et al. (2008) for human healthy cells and corresponding abnormal carcinoma of the lung, 
estimated about $2100 \mathrm{~Pa}$ and $560 \mathrm{~Pa}$, respectively. In this case, setting $\nu=0.4$, the stiffer value associated to the healthy cells can be obtained through the proposed model by assuming a prestretch $\lambda_{p}=1.32$ and considering $n=75$ active protein filaments, the cancer cell elastic modulus being caught by merely reducing to about 26 the number of active filaments to simulate possible lower levels of polymerization in the cytoskeleton structure of cancer cells to facilitate squeezing and metastatic migration abilities.

\section{In-frequency response of adherent single-cell viscoelastic systems}

\section{incorporating cytoskeleton prestress}

By exploiting the results obtained in the previous Sections with reference to the effects of the prestretched/prestressed cytoskeleton filaments on the cell stiffness and by starting from an approach proposed by Or and Kimmel (2009) and recently further developed by Fraldi et al. (2015) to analyze the case of vibrating cell nucleus in a viscoelastic environment excited by Low Intensity Therapeutic Ultra-Sound (LITUS), let us consider a single-cell system whose dynamics is reduced to an oscillating mass immersed in a viscoelastic medium (see Figure 5). To represent the nucleus, a rigid sphere of radius $R$ is thus considered, by ideally concentrating in it the entire mass of the cell and modeling the environment as a homogeneous and isotropic viscoelastic medium. Under these hypotheses, the whole cell dynamics is completely governed by one degree of freedom stimulated by a velocity law taken in the form

$$
v_{m}(t)=v_{m 0} e^{-i \omega_{0} t}
$$

where $v_{m}$ represents the velocity prescribed to the medium, $v_{m 0}$ is the amplitude of the complex velocity phasor, with $\omega_{0}=2 \pi f$ denoting the oscillations angular frequency and $f$ the measured frequency in Hz. Accordingly to the above cited Literature, the motion can be described by the equation

$$
f_{m}=m_{o b} a_{o b}=\frac{4}{3} \pi R^{3} \rho_{o b} \frac{d^{2} u_{o b}}{d t^{2}}=f_{a c}-f_{\text {res }}
$$

In eq. (18), $t$ is the time, $f_{m}$ is the inertial force, $m_{o b}$ represents the nucleus mass of density $\rho_{o b}, u_{o b}$ is the displacement and the driving force that is kindled by the acoustic pressure gradients triggered by the ultrasound transducer in the system is $f_{a c}$. It is worth to highlight that -in the case under analysis- 
the object is very small if compared with the acoustic wavelength and thus the form of the acoustic force can be simply reduced to a force which ideally would act on a sphere of the same radius, in absence of the object (Maxey and Riley (1983)); this permits to write

$$
f_{a c}=\frac{4}{3} \pi \rho_{m} R^{3} \frac{D v_{m}}{D t} \equiv \frac{4}{3} \pi \rho_{m} R^{3} \frac{d v_{m}}{d t}
$$

where $\rho_{m}$ is the density of the medium. Additionally, dimensional analyses show that the convective term is small and, as a result, the absence of spatial variability allows to adopt in eq. (19) timedifferentiation $d / d t$ instead of the substantial derivative $D / D t$ (Or and Kimmel (2009)). It has to be also noticed that $f_{\text {res }}$ represents the response force applied to the object by its surrounding, due to their relative motion, and thus it will depend on the overall rheological features of the environment. In order to catch possible further insights on the single-cell behavior, this force incorporates, in a parametric way, key geometrical and mechanical properties of interest.

The analyses will be performed by first utilizing two quasi-standard viscoelastic models (Voigt and Maxwell), then introducing a generalized standard linear Kelvin model in which dashpot and spring elements are replaced by so-called Spring-Pot systems, in different ways recently used to describe with success the mechanical behavior of biological materials (Deseri et al. (2013)).

Additionally, by recalling the well-known relationship between Laplace and Fourier transforms (i.e. $\left.\mathscr{F}[\cdot]=\left.\mathscr{L}[\cdot]\right|_{s=i \omega}\right)$, the Laplace transform is utilized in what follows to solve the differential problem at hand, in this manner obtaining the response of the systems directly in terms of frequency (Fraldi et al. (2015)).

With respect to the assigned initial conditions, in all the simulations the single-cell system is assumed to be initially at rest, that is

$$
\left.u_{o b}\right|_{t=0}=0 \quad,\left.\quad \frac{d u_{o b}}{d t}\right|_{t=0}=0
$$

By therefore Laplace transforming the eq. (18), one finally obtains

$$
F_{m}=\frac{4}{3} \pi R^{3} \rho_{o b} s^{2} U_{o b}=F_{a c}-F_{r e s}
$$

in which $s$ denotes the Laplace variable and all the (Laplace-)transformed terms are indicated with capital letters. Accordingly, $F_{a c}$ that appears in eq. (21) represents the Laplace transforming of the 
acoustic force $f_{a c}$ reported in the eq. (19), so obtaining

$$
F_{a c}=\frac{4}{3} \pi \rho_{m} R^{3} s V_{m}=\frac{4}{3} \pi \rho_{m} R^{3} s^{2} U_{m}
$$

The reader can find some further details of the viscoelastic models presented below by making reference to Fraldi et al (Fraldi et al. (2015)).

\section{Enhanced Voigt and Maxwell models for single-cell}

As known, the Voigt idealization assumes that viscous and elastic elements are placed in parallel to each other (see Figure 5p. In this way, the resulting overall force can be determined by the simple sum of the forces due the the single constituting elements as

$$
f_{\text {res }}=f_{\mu}+f_{G}
$$

where $f_{\mu}$ represents the viscous contribution and $f_{G}$ the elastic response. In case of rigid object rapidly vibrating in a viscous fluids, the viscous term can be helpfully represented with the following explicit form, as suggested by Basset (1888), Landau and Lifshitz (1987) and Or and Kimmel (2009)

$$
f_{\mu}=6 \pi R \mu\left(1+\sqrt{\frac{\omega R^{2}}{2 \eta}}\right)\left(v_{o b}-v_{m}\right)+\frac{2}{3 p} \pi R^{3} \rho_{m}\left(1+\frac{9 p}{2} \sqrt{\frac{2 \eta}{\omega R^{2}}}\right)\left(\dot{v}_{o b}-\dot{v}_{m}\right)
$$

with $\eta$ and $\mu$ kinematic and dynamic medium viscosities, respectively, and $v=\dot{u}$ the velocity. Note that, in eq. (24), the structure of the viscous force is different from the standard Stokes one,

frequency-dependent terms as well as an inertial (spurious) contribution $3 \pi R^{3} \rho_{m} \sqrt{\frac{2 \eta}{\omega R^{2}}}$, named added mass(Brennen (1982)), additionally appearing, $p$ (here is $p=2$ ) being the number of elements in parallel ad hoc introduced in Fraldi et al. (2015) to solve an ambiguous situation already pointed out by Or and Kimmel (2009). In fact, as already highlighted in Fraldi et al. (2015), to avoid contrived solutions to remove "the excessive added-mass term" that "erroneously twice appears" in Or and Kimmel (2009), the viscoelastic forces are here set to obtain that any combined viscoelastic scheme derived from the general fractional-based SLK model contains the sole added-mass and virtual friction contributions to be considered. 
Following Ilinskii et al. (2005), the elastic force $f_{G}$ can be given by

$$
f_{G}=6 \pi G R\left(u_{o b}-u_{m}\right)+6 \pi R^{2} \sqrt{G \rho_{m}}\left(\dot{u}_{o b}-\dot{u}_{m}\right)+\frac{2}{3 p} \pi R^{3} \rho_{m}\left(\ddot{u}_{o b}-\ddot{u}_{m}\right)
$$

Similarly to the viscous force, the elastic contribution in $(25)$ is presented in a somehow enhanced version with respect to the standard Hooke law, to include key effects of the cell nucleus-environment dynamic interactions which characterize the actual physical behavior of the overall system due to rapid fluctuations. In eq. (25), the physics is then caught through two additional dissipative and inertial terms, respectively equal to $6 \pi R^{2} \sqrt{G \rho_{m}}$ (the virtual friction) and the so-called added mass, $G$ representing the first Lamé modulus of and $u_{m}$ the vibrational displacement of the medium. Also, for convenience, it is assumed that

$$
\begin{gathered}
c_{0 G}=6 \pi G R, \quad c_{1 G}=6 \pi R^{2} \sqrt{G \rho_{m}}, \quad c_{2 G}=\frac{2}{3 p} \pi R^{3} \rho_{m} \\
c_{1 \mu}=6 \pi R \mu\left(1+\sqrt{\frac{\omega R^{2}}{2 \eta}}\right), \quad c_{2 \mu}=\frac{2}{3 p} \pi R^{3} \rho_{m}\left(1+\frac{9 p}{2} \sqrt{\frac{2 \eta}{\omega R^{2}}}\right)
\end{gathered}
$$

a further dimensionless parameter being

$$
\zeta=\frac{\rho_{o b}}{\rho_{m}}=\frac{1}{1+\gamma}
$$

where $\gamma=\rho_{m} \rho_{o b}^{-1}-1$. At the end, the modified Voigt viscoelastic equation is obtained as

$$
f_{\text {res }}=c_{0 G}\left(u_{o b}-u_{m}\right)+\left(c_{1 \mu}+c_{1 G}\right)\left(\dot{u}_{o b}-\dot{u}_{m}\right)+\left(c_{2 \mu}+c_{2 G}\right)\left(\ddot{u}_{o b}-\ddot{u}_{m}\right)
$$

from which, by Laplace transforming, one finally has

$$
F_{r e s}=\left(U_{o b}-U_{m}\right)\left[c_{0 G}+\left(c_{1 \mu}+c_{1 G}\right) s+\left(c_{2 \mu}+c_{2 G}\right) s^{2}\right]
$$

and, replacing (30) and (22) in (21) and after some algebraic passages, the following equation is found

$$
\left[c_{0 G}+\left(c_{1 \mu}+c_{1 G}\right) s+\left(\left(c_{2 \mu}+c_{2 G}\right)+\frac{4}{3} \pi \rho_{o b} R^{3}\right) s^{2}\right] \Delta U=\frac{4}{3} \pi \gamma \rho_{o b} R^{3} s V_{m}
$$


where $\Delta U=U_{o b}-U_{m}$. By solving the eq. (31), the analytical solution of the the in-frequency response of the system -in terms of relative displacement $\Delta U$ of the cell nucleus with respect to the envinronment- is obtained in the form

$$
\left.|\Delta U|\right|_{s=i \omega}=\left.\left|\frac{\frac{4}{3} \pi \gamma \zeta \rho_{m} R^{3} s V_{m}}{c_{0 G}+\left(c_{1 \mu}+c_{1 G}\right) s+\left(\left(c_{2 \mu}+c_{2 G}\right)+\frac{4}{3} \pi \rho_{o b} R^{3}\right) s^{2}}\right|\right|_{s=i \omega}
$$

Complementary to the case of Voigt, Maxwell systems present elastic and viscous elements ideally placed in series (see Figure 57. The overall response is therefore found by imposing the following isostress condition

$$
F_{G}=F_{\mu}=F_{r e s}
$$

thus equating the sum of the viscous and the elastic displacement contributions to the total relative displacement, that is

$$
\Delta U=\Delta U_{G}+\Delta U_{\mu}
$$

where $F_{G}$ and $F_{\mu}$ represent the Laplace transforms of the elastic and viscous forces respectively given in eqs. (25) and (24), so obtaining

$$
F_{G}=\left(c_{0 G}+c_{1 G} s+c_{2 G} s^{2}\right) \Delta U_{G}, \quad F_{\mu}=\left(c_{1 \mu} s+c_{2 \mu} s^{2}\right) \Delta U_{\mu}
$$

from which the elastic and viscous displacements can be explicitly written as

$$
\Delta U_{G}=\frac{F_{G}}{c_{0 G}+c_{1 G} s+c_{2 G} s^{2}}, \quad \Delta U_{\mu}=\frac{F_{\mu}}{c_{1 \mu} s+c_{2 \mu} s^{2}}
$$

By recalling $F_{\text {res }}$ from eq. (21) and considering eq. (36), the closed-form solution for the in frequency response of the enhanced Maxwell system is found as 


$$
\left.|\Delta U|\right|_{s=i \omega}=\left.\left|\frac{\frac{4}{3} \pi \gamma \rho_{o b} R^{3} s V_{m}}{1+\frac{4}{3} \pi \rho_{o b} R^{3} s^{2}\left(\frac{1}{c_{1 \mu} s+c_{2 \mu} s^{2}}+\frac{1}{c_{0 G}+c_{1 G} s+c_{2 G} s^{2}}\right)}\right|\right|_{s=i \omega}
$$

\section{Single-cell response through generalized fractional derivative-based Standard Linear Kelvin paradigms}

\section{Added mass and virtual friction in Spring-Pot models}

In a viscoelastic medium employing Fractional Derivatives, the mechanical behavior is interpreted through the introduction of so-called Spring-Pot systems. The first time that the fractional derivative concept is traced in an epistolary correspondence between de L'Hospital and Leibniz dated back to 1695, where they tried to give an answer to the question: "What does the derivative $d^{n} f(x) / d x^{n}$ mean if $n=1 / 2$ ?". From that time, a new branch of mathematics -fractional calculus, a generalization of the commonly used integer-order differentiation and integration- has been formally developed. The basic idea, as suggested by Riemann-Liouville, is in fact to interpret the fractional derivative as the inverse operation of a fractional integral. The use of fractional derivatives in viscoelasticity can be traced in the work by Nutting (1921), where, from the best fitting of experimental curves, he noted the possibility of describing the relationship between deformation and time through a power law, i.e. $u \propto t^{n} F^{m}$, in which $F$ is the force and $u$ represents the displacement. Successively, in 1949, Blair and Caffyn (1949) analytically justified this experimental curve by means of the fractional derivatives, also introducing the Spring-Pot model. Afterwards, Caputo (1969) in detail proposed a fractional derivative operator, namely ${ }_{a}^{C} D_{t}^{\alpha}$, which could be used in "real world":

$$
{ }_{a}^{C} D_{t}^{\alpha} f(t)=\frac{1}{\Gamma(n-a)} \int_{a}^{t}(t-s)^{n-\alpha-1} f^{n}(s) d s \quad \forall \quad n-1 \leq \alpha \leq n
$$

$\Gamma$ representing the Euler Gamma function and $f(t)$ being integrable in $[a, t]$.

In the last years, several scientific papers involving fractional calculus-based viscoelasticity has been presented, for both approaching standard problems and analysing complex systems in pioneering physical and engineering fields (see for example Atanackovic et al. (2007); Bagley (1983); Deseri et al. (2013, 2014); Di Paola et al. (2009, 2013); Grillo et al. (2015); Mainardi (2012); Metzler and 
Klafter (2000); Schiessel and Blumen (1993)). For the present purpose, however, the Spring-Pot model is essentially that firstly proposed by Blair and Caffyn (1949), here ad hoc generalized to integrate added mass and virtual friction as additional system features. As a consequence, the following definition of the Spring-Pot response force $f_{S P}$ is introduced

$$
f_{S P}:=C_{\alpha}\left(\begin{array}{l}
C \\
0
\end{array} D_{t}^{\alpha}\left(u_{o b}-u_{m}\right)\right)+c_{1 S P}\left(\dot{u}_{o b}-\dot{u}_{m}\right)+c_{2 S P}\left(\ddot{u}_{o b}-\ddot{u}_{m}\right)
$$

in which ${ }_{0}^{C} D_{t}^{\alpha}$ represents the Caputo's fractional time-derivative of order $\alpha(\alpha \in[0,1]$ being over the time interval $(0, t))$ and $C_{\alpha}$ is a suitable frequency-dependent parameter written by starting from Koeller (1984) as follows

$$
C_{\alpha}=c_{0 G}\left(\frac{c_{1 \mu}}{c_{0 G}}\right)^{\alpha}
$$

In particular, dissipative and inertial terms were incorporated in the model by postulating the simplest mathematical structure

$$
c_{1 S P}=(1-\alpha) c_{1 G}, \quad c_{2 S P}=c_{2 G}\left(1+\alpha \frac{9 p}{2} \sqrt{\frac{2 \eta}{\omega R^{2}}}\right)
$$

in this manner reproducing the elastic and viscous models proposed by Or and Kimmel (2009) as special limit cases, that is $\alpha=0$ and $\alpha=1$, respectively.

At the end, by substituting eq. (39) into eq. (21) and invoking the fractional derivative rule allowing to Laplace transform by preserving the classical (integer) derivative law for the Laplace variable $s$, namely ${ }_{0}^{C} D_{t}^{\alpha} \stackrel{\mathscr{L}}{\rightarrow} s^{\alpha}$, the in-frequency response of the spring-pot model, in terms of nucleusenvironment relative displacement, can be finally found in the following form

$$
\forall \alpha \in[0,1],\left.|\Delta U|\right|_{s=i \omega}=\left.\left|\frac{\frac{4}{3} \pi \gamma \rho_{o b} R^{3} s V_{m}}{\left(\frac{4}{3} \pi \rho_{o b} R^{3}+c_{2 S P}\right) s^{2}+c_{1 S P} s+C_{\alpha} s^{\alpha}}\right|\right|_{s=i \omega}
$$

\section{Generalized SLK model incorporating Spring-Pot systems}

To enrich Maxwell and Voigt viscoelastic behaviors, different so-called Standard Linear Solid (SLS) systems can be encountered in the Literature (Tschoegl (1989)). Among these, one of the most uti- 
lized scheme is constituted by the Standard Linear Kelvin (SLK) model, obtained by placing in series an elastic spring with a Voigt system, the so-called Maxwell-Wiechert model representing an alternative -and somehow complementary-configuration where an elastic spring is positioned in parallel with a Maxwell system, whose multi-element version leads to the well-known Prony series method. Given that the Spring-Pot might be physically interpreted as a viscoelastic system intrinsically capable to smoothly generate intermediate mechanical responses as the above mentioned parameter $\alpha$ moves from zero to one, in the limit cases respectively giving purely elastic and viscous behaviors, a possible generalization of the SLK model can be envisaged by replacing in it each dashpot and spring with a spring-pot, as shown in Figure 5, by furthermore enhancing the resulting model by suitably including added mass and virtual friction terms. In this way, a powerful (linear) low-parameter viscoelastic system can be realized with the important advantage that, by essentially following the above proposed strategies, analytical solutions of the corresponding in-frequency system response can be always derived for any modulation and combination of the spring-pot parameter $\alpha$. As a result, a wide range of otherwise unforeseeable viscoelstaic responses can be caught and all the simpler viscoelastic models -and the related analytical solutions- recalled above, including the Or and Kimmel (2009) ones, can be obtained as special limit cases of the proposed generalized SLK scheme as well. With reference to the configuration of the spring-pot in the proposed generalized SLK viscoelastic system (see Figure 5), let us write equilibrium among forces and compatibility for the displacements as

$$
\begin{gathered}
f_{S L K}=f_{P}=f_{S P 3} \\
\Delta u_{S L K}=\Delta u_{P}+\Delta u_{S P 3}
\end{gathered}
$$

in which $f_{S L K}$ represents the total force, $f_{P}=f_{S P 1}+f_{S P 2}, f_{S P 1}, f_{S P 2}$ and $f_{S P 3}$ being the single contributions given by the three spring-pots whose explicit forms are furnished by the eqn (39) and the displacement $\Delta u_{P}=\Delta u_{S P 1}=\Delta u_{S P 2}$.

By Laplace transforming $f_{P}$ and $f_{S P 3}$, fractional derivative rule ${ }_{0}^{C} D_{t}^{\alpha} \stackrel{\mathscr{L}}{\rightarrow} s^{\alpha}$ gives

$$
\begin{gathered}
F_{P}=\left[C_{\alpha 1} s^{\alpha 1}+C_{\alpha 2} s^{\alpha 2}+\left(c_{1 S P 1}+c_{1 S P 2}\right) s+\left(c_{2 S P 1}+c_{2 S P 2}\right) s^{2}\right] \Delta U_{P} \\
F_{S P 3}=\left[C_{\alpha 3} s^{\alpha 3}+c_{1 S P 3} s+c_{2 S P 3} s^{2}\right] \Delta U_{S P 3}
\end{gathered}
$$


Algebraic manipulations allow to write

$$
\begin{gathered}
\Delta U_{P}=\frac{F_{P}}{C_{\alpha 1} s^{\alpha 1}+C_{\alpha 2} s^{\alpha 2}+\left(c_{1 S P 1}+c_{1 S P 2}\right) s+\left(c_{2 S P 1}+c_{2 S P 2}\right) s^{2}} \\
\Delta U_{S P 3}=\frac{F_{S P 3}}{C_{\alpha 3} s^{\alpha 3}+c_{1 S P 3} s+c_{2 S P 3} s^{2}}
\end{gathered}
$$

and therefore, by recalling the previously obtained relationship for $F_{r e s}$, the closed-form in-frequency solution for the SLK system is finally determined as

$$
\left.\left|\Delta U_{S L K}\right|\right|_{s=i \omega}=\left.\left|\frac{\frac{4}{3} \pi \gamma \rho_{o b} R^{3} s V_{m}\left(\frac{1}{s\left(c_{1 S P 1}+c_{1 S P 2}\right)+s^{2}\left(c_{2 S P 1}+c_{2 S P 2}\right)+C_{\alpha 1} s^{\alpha 1}+C_{\alpha 2} s^{\alpha 2}}+\frac{1}{s\left(c_{1 S P 3}+c_{2 S P 3} s\right) C_{\alpha 3} s^{\alpha 3}}\right)}{\frac{4}{3} \pi \rho_{o b} R^{3} s^{2}\left(\frac{1}{s\left(c_{1 S P 1}+c_{1 S P 2}\right)+s^{2}\left(c_{2 S P 1}+c_{2 S P 2}\right)+C_{\alpha 1} s^{\alpha 1}+C_{\alpha 2} s^{\alpha 2}}+\frac{1}{s\left(c_{1 S P 3}+c_{2 S P 3} s\right)+C_{\alpha 3} s^{\alpha 3}}\right)-1}\right|\right|_{s=i \omega}
$$

This equation gives thus the analytical response of a generalized SLK model capable to cover a wide ranges of possible intermediate viscoelastic behaviors reproduced by tuning the fractional derivative order $\alpha$ characterizing the spring-pots, also incorporating added mass and virtual friction effects (Fraldi et al. (2015)) and replicating -as limit cases- all the simpler (viscous and elastic) as well as non-standard single-cell literature models (Or and Kimmel (2009)). Some systems, obtained by modulating the parameters $\alpha$ in the proposed model, are summarized in Table 1 with reference to selected cases afterwards utilized for simulating the in-frequency response of single cells to mechanical loads, say Elastic, Viscous, Voigt (V), Maxwell (M) and standard linear Kelvin (SLK) limit cases, as well as the three intermediate chosen configurations, say SLK_1, SLK_2 and SLK_3.

\section{The resonance hypothesis in adherent cells: the role of prestretch and number of active cytoskeleton filaments}

In a recent paper by some of the present authors (Fraldi et al. (2015)) the in-frequency response of single-cell systems has been in detail analyzed through simple (one-degree of freedom) visco-elastic schemes, by also conducting sensitivity analyses aimed to gain information about positioning and magnitude of the response peaks for envisaging possibilities of exploiting the stiffness discrepancies experimentally observed between healthy and tumor cells for mechanically targeting and selectively 
attacking cancer cells. To make this, the above recalled authors explored the in-frequency responses of a wide class of viscoelastic single-cell paradigms, by ad hoc introducing a new generalized fractional derivative-based SLK model and constructing the related analytical solutions, whose results were referred to ranges of mechanical properties and physical parameters actually measured at singlecell level and reported in the consolidated literature.

However, when dealing with living systems, the measures of stiffness can be significantly affected by intrinsic structural changes of the biological matter, for example by the reorganization dynamics guided by polymerization-depolymerization processes which change the internal configuration of the cytoskeleton, so regulating adhesion and migration cell capabilities and in turn provoking non homogeneous cell deformations and changes in stiffness (Bao and Suresh (2003); Brunner et al. (2009); Rodriguez et al. (2013)), with Young's moduli also oscillating from about $100 \mathrm{~Pa}$ to $10 \mathrm{kPa}$ (Caille et al. (2002); Cross et al. (2008, 2007); Faria et al. (2008); Ketene et al. (2012); Lekka et al. (2012a, 1999. 2012b); Li et al. (2008); Nikkhah et al. (2010); Prabhune et al. (2012); Rebelo et al. (2013)).

Nevertheless, the vast majority of the experimental data somewhat considers stiffness of "round" (suspended) cells and -at the best authors knowledge- no many efforts have been devoted, from the modeling standpoint, to mechanically relate the overall change of cell stiffness to its stretched configuration, as well as to the average number of active/assembled cytoskeletal filaments.

Therefore, by starting from the literature experimental results and taking into account the ranges within which actual measured mechanical features of cells can oscillate, the overall stiffness -determined from the proposed elemental non-linear elastic single-cell model- has been introduced into the fractional derivative-based SLK scheme. In this way, the cell visco-elastic behavior explicitly depends, among other geometrical and physical parameters, upon the stiffness resulting from the number of active cytoskeletal filaments, their prestretch level due to possible adherent configurations, as well as from the round shape-associated cell Young modulus, directly related to the cell line and to the cell (i.e. healthy or cancer) state.

To highlight the possibility of following the above described strategy for representing the whole range of the experimentally measured single-cell mechanical properties, in both suspended and adherent conditions, also demonstrating that viscoelaatic response peaks still fall within frequencies intervals of ultrasound which would still preserve the possibility of of selectively inducing resonance-like phenomena in cells (Fraldi et al. (2015)), sensitivity analyses have been thus ad hoc performed by making 
variable the overall intrinsic round-shaped cell Young modulus, the prestretch and the number of active micro-filaments, the cytosol viscosity and the nucleus size being prescribed and set equal to average values, in this manner covering the entire range of the mechanical data given in the literature for many cell lines investigated.

By essentially following data and methods already introduced in Fraldi et al. (2015) and with respect to the notations proposed for the generalized SLK visco-elastic systems, the analyses reported below have been conducted by assuming medium vibration velocity magnitude $v_{m 0}=0.12 \mathrm{~ms}^{-1}$, determined in case of plain progressive waves characterized by acoustic intensity of $1 \mathrm{~W} \mathrm{~cm}^{-2}$ and associated intensity $I=0.5 \rho_{m} c v_{m 0}^{2}, c=1500 \mathrm{~ms}^{-1}$ being the speed of sound at room temperature (Lide et al. (2008)) at which mass density of the medium has been also assumed to be coincident with that of the water, the nucleus -as reported in (Michelet-Habchi et al.(2005))- being considered about 30\% more dense than the environment.

More specifically, the performed analyses have been referred to six selected viscoelastic schemes, that is the enhanced Voigt, Maxwell and SLK ones and further three generalized fractional derivativebased SLK models constructed by positioning in the sections 1, 2 and 3 spring-pots with $\alpha=0.5$ (see Figure 5), in all the cases also taking into account added mass and virtual friction effects. Additionally, the elastic modulus $G$ appearing in the fractional derivative-based models implicitly takes into account the cell configuration (suspended and adherent) through prestretch and number of active filaments determined by eqs. (13) and (16). The theoretical outcomes have been carried out by making the calculations using the symbolic commercial code Wolfram Mathematica ${ }^{\circledR}$ (Wolfram (2003)) and the results have been represented in the domain of the frequencies within the interval most interesting for biomedical applications, i.e. $1 \mathrm{kHz} \leq f \leq 100 \mathrm{MHz}$. In particular, the main attention is paid to the in-frequency system responses plotted in terms of maximum relative displacement $|\Delta U|$, in the time domain representing the magnitude of relative oscillations between environment and cell nucleus, induced by ultrasound.

Figures 6, 7 and 8 collect the most relevant results from the analytical models: therein, cell stiffness, prestrech intensity and number of filaments have been assumed to vary within intervals compatible with experimental findings, keeping fixed the other complementary parameters and choosing for them the most common literature values, i.e. Young modulus $E=2100 \mathrm{~Pa}$ (Cross et al. (2007)), cell nucleus radius $R=1 \mu \mathrm{m}($ Cowin and Doty $(2007))$ and viscosity of the water $\mu=10^{-3} \mathrm{~Pa} \times \mathrm{s}($ Or and 
Kimmel (2009)).

Figure 6 in particular illustrates the cell in-frequency response in terms of relative displacement, by parametrically making variable the cell stiffness from $E=100 \mathrm{~Pa}$ to $E=10 \mathrm{kPa}$, coherently with data ranges reported in the experimental literature. Similarly, Figures 7 and 8 show the results for the six viscoelastic models chosen, by plotting again displacement amplitude against frequency and respectively setting the tangent Young modulus $\left(E=2.6 \times 10^{9} \mathrm{~Pa}\right)$ and Poisson ratio $(\nu=0.4)$ of the microfilamts, the viscosity $\mu=10^{-3} \mathrm{~Pa} \times \mathrm{s}$ and making variable the prestretch $\lambda_{p}$ and the number $n$ of cytoskeleton filaments.

The outcomes obtained from the sensitivity analyses confirm both qualitative trends and quantitative results already found in Fraldi et al. (2015), with growing peak frequencies and associated decreasing displacement amplitudes as the overall cell stiffness grows up as a consequence of the increase of the intrinsic Young moduli of the (round) cells (see Figure 6), as well as when the cell stiffening is induced by its adherent configuration, a situation here modeled by increasing the tensile pre-stresses in the cytoskeletal elements and the number of prestretched filaments (see Figures 7 and 8). Also, in all the analyzed single-cell systems, the results highlight that the maximum vibrations $|\Delta U|$ and associated peak frequencies always fall within the interval $10^{4} \sim 10^{6} \mathrm{~Hz}$, a range coherent with that experimentally established by several works (see, for instance, Lejbkowicz and Salzberg (1997) and Johns (2002)) that still authorizes -at least in principle- to think of obtaining resonance-like responses by stimulating single cells by means of ultrasounds. Importantly, for all the viscoelastic schemes, the obtained results confirm that US-induced mechanical vibrations, $|\Delta U|$, are mostly comparable (or greater than) spontaneous thermal fluctuations if both calculated in limit situations of pure elastic media -where MSD (the Mean Square Displacement) is $\left\langle u_{T, e}^{2}\right\rangle=\frac{k_{B} T}{\pi R G}($ Ohshima and Nishio (2001))and pure viscous systems, where MRD (the Mean Relaxation Distance) is $\left\langle u_{T, \eta}\right\rangle=\frac{2 R^{2} \rho_{o b} v_{0}}{9 \mu}$ (Kittel and Krocmer (1980)), $k_{B}$ being the Botzmann constant, $T$ the absolute temperature and $v_{0}$ the initial velocity. It can be in fact numerically verified that the MSD maximum square root is of the order of $10^{-9} \mathrm{~m}$ while MRD can oscillate between $10^{-15} \mathrm{~m}$ and $10^{-8} \mathrm{~m}$, in both the cases leading to values smaller than (or at most comparable with) the vibration amplitude peaks obtained theoretically from the above mentioned parametric analyses (see Figures 6, 7, and 8). 


\section{Conclusions and future challenges}

By following a very recent work by some of the authors aimed to analyze the in-frequency response of single-cell systems to mechanical stimuli (Fraldi et al. (2015)), a new enhanced fractional derivativebased viscoelastic scheme incorporating the non-linear elastic behavior of the cell cytoskeleton has been here proposed by first introducing an elemental sub-cellular structural model, then following -after a bottom-up procedure to meet the microscale- a small-on-large approach to study the dynamics (nucleus vibration) of the cell as a whole. In this way, together the geometrical and physical parameters usually involved in the mechanical study of suspended (round) cells, some further key factors influencing the overall stiffness of adherent cells have been taken into account, that is the prestress/prestretch and the average number of attending protein filaments. Actually, the cell stiffness can significantly change if, by interacting with the ECM, the focal adhesion points invite cell cytoskeleton to assume deformed configurations. Stretching and collective reconfigurations of protein filaments guided by polymerization/depolymerization processes generally accompany the transition of a cell from a suspended to an adherent state. Forces and srains in the prestretched stress fibers thus play a crucial role in the dynamic updating of the mechanical properties of single cells, as well as in determing their viscoelastic response to mechanical stimuli. In this framework, it has been indeed experimentally shown that at low-frequency cyclic loads and strain regimes from $\left(10^{-2}\right.$ to $\left.10^{-1}\right)$, can actually generate structural alterations or physical rupture of cytoskeletal elements in living cells (Or and Kimmel (2009)). Similar effects have been also observed at relatively high frequencies, in particular for the case of cells stimulated by ultrasound, as found in Mizrahi et al. (2012) and Lejbkowicz and Salzberg (1997) which have shown that configurational and mechanical changes were caused at ultrasonic frequencies $\left(10^{6} \mathrm{~Hz}\right)$ by very small strains $\left(10^{-5}\right)$ as well as at physiological frequencies $\left(10^{0} \mathrm{~Hz}\right)$ by relatively large strains $\left(10^{-1}\right)$.

With reference to the obtained theoretical findings, a rough value of the axial elongation equivalent to a simple uniaxial strain can be roughly estimated as $\varepsilon \propto \frac{|\Delta U|}{(10 \times R)}$ : as a consequence, by recalling that a typical size of a cell nucleus can be found in the interval $\left(2 \times 10^{-7}, 10^{-5}\right) \mathrm{m}$ while vibration magnitude are obtained from about $10^{-9} \mathrm{~m}$ to $10^{-7} \mathrm{~m}$, strains from $10^{-5}$ up to $10^{-1}$ can be determined and higher values can be hence reached due to the prestretch imprisoned in the cytoskeleton filaments of adherent cells.

Also, the peak frequencies, derived from the theory by using the parametric fractional calculus-based 
viscoelastic schemes, span from tens $\mathrm{kHz}$ to about one $\mathrm{MHz}$, these limit values being both experimentally recognized as critical frequencies at which cells exhibit significant biological configurational alterations due to mechanical effects which prevail on thermal ones (Johns (2002); Schuster et al. (2013)).

At least in principle, after a few seconds of exposure to ultrasound (e.g. LITUS), cell structural modifications or failure due to cyclic loading and associated fatigue phenomena could be thus actually expected and this, on the basis of the ascertained fact that cancer cells are found to be always softer than their normal (healthy) counterparts -independently from the cell line- might pave the way for designing new mechanically-based tumor markers and strategies to selectively attack cancer cells.

\section{References}

Atanackovic, T. M., Pilipovic, S., and Zorica, D. (2007). A diffusion wave equation with two fractional derivatives of different order. Journal of Physics A: Mathematical and Theoretical, 40(20):5319-5333.

Bagley, R. L. (1983). A Theoretical Basis for the Application of Fractional Calculus to Viscoelasticity. Journal of Rheology, 27(3):201.

Bao, G. and Suresh, S. (2003). Cell and molecular mechanics of biological materials. Nature materials, 2(11):715-725.

Basset, A. B. (1888). A Treatise on Hydrodynamics, with numerous examples. Cambridge.

Blair, G. S. and Caffyn, J. (1949). An application of the theory of quasi-properties to the treatment of anomalous strain-stress relations. The London, Edinburgh, and Dublin Philosophical Magazine and Journal of Science, 40(300):80-94.

Brennen, C. (1982). A review of added mass and fluid inertial forces. Technical Report January.

Brunner, C., Niendorf, A., and Käs, J. a. (2009). Passive and active single-cell biomechanics: a new perspective in cancer diagnosis. Soft Matter, 5(11):2171. 
Caille, N., Thoumine, O., Tardy, Y., and Meister, J.-J. (2002). Contribution of the nucleus to the mechanical properties of endothelial cells. Journal of Biomechanics, 35(2):177-187.

Caputo, M. (1969). Elasticità e dissipazione. Zanichelli, Bologna.

Chen, Q. and Pugno, N. M. (2013). Bio-mimetic mechanisms of natural hierarchical materials: A review. Journal of the Mechanical Behavior of Biomedical Materials, 19:3-33.

Chumakova, O. V., Liopo, A. V., Evers, B. M., and Esenaliev, R. O. (2006). Effect of 5-fluorouracil, Optison and ultrasound on MCF-7 cell viability. Ultrasound in medicine \& biology, 32(5):751-8.

Cowin, S. C. and Doty, S. B. (2007). Tissue mechanics. Springer New York.

Cross, S., Jin, Y.-S., Tondre, J., Wong, R., Rao, J., and Gimzewski, J. (2008). AFM-based analysis of human metastatic cancer cells. Nanotechnology, 19(38):384003.

Cross, S. E., Jin, Y.-S., Rao, J., and Gimzewski, J. K. (2007). Nanomechanical analysis of cells from cancer patients. Nature nanotechnology, 2(12):780-783.

Del Piero, G. and Deseri, L. (1997). On The Concepts of State and Free Energy in Linear Viscoelasticity. Archive for Rational Mechanics and Analysis, 138(1):1-35.

Delsanto, P. P., Condat, C. A., Pugno, N., Gliozzi, A. S., and Griffa, M. (2008). A multilevel approach to cancer growth modeling. Journal of Theoretical Biology, 250:16-24.

Deseri, L., Fabrizio, M., and Golden, M. (2006). The concept of a minimal state in viscoelasticity: New free energies and applications to PDEs. Archive for Rational Mechanics and Analysis, 181:4396.

Deseri, L., Paola, M. D., Zingales, M., and Pollaci, P. (2013). Power-law hereditariness of hierarchical fractal bones. International Journal for Numerical Methods in Biomedical Engineering, 29:13381360.

Deseri, L., Zingales, M., and Pollaci, P. (2014). The state of fractional hereditary materials (FHM) . Discrete and continuos dynamical systems series B, 19(7):2065-2089. 
Di Paola, M., Marino, F., and Zingales, M. (2009). A generalized model of elastic foundation based on long-range interactions: Integral and fractional model. International Journal of Solids and Structures, 46(17):3124-3137.

Di Paola, M., Pinnola, F. P., and Zingales, M. (2013). A discrete mechanical model of fractional hereditary materials. Meccanica, 48(7):1573-1586.

DuFort, C. C., Paszek, M. J., and Weaver, V. M. (2011). Balancing forces: architectural control of mechanotransduction. Nature reviews. Molecular cell biology, 12(5):308-319.

Ellwart, J. W., Brettel, H., and Kober, L. O. (1988). Cell membrane damage by ultrasound at different cell concentrations. Ultrasound in medicine \& biology, 14(1):43-50.

Faria, E. C., Ma, N., Gazi, E., Gardner, P., Brown, M., Clarke, N. W., and Snook, R. D. (2008). Measurement of elastic properties of prostate cancer cells using AFM. The Analyst, 133(11):14981500.

Fraldi, M. (2014). The mechanichal beauty of hierarchically organized living structures. Scienza \& Filosofia, 11:13-24.

Fraldi, M. and Cowin, S. C. (2004). Inhomogeneous elastostatic problem solutions constructed from stress-associated homogeneous solutions. Journal of the Mechanics and Physics of Solids, 52:2207-2233.

Fraldi, M., Cugno, A., Deseri, L., Dayal, K., and Pugno, N. M. (2015). A frequency-based hypothesis for mechanically targeting and selectively attacking cancer cells. Journal of The Royal Society Interface, 12(111):20150656.

Grillo, G., Muratori, M., and Punzo, F. (2015). On the asymptotic behaviour of solutions to the fractional porous medium equation with variable density. Discrete and Continuous Dynamical Systems, 35(12):5927-5962.

Guiot, C., Pugno, N., and Delsanto, P. P. (2006). Elastomechanical model of tumor invasion. Applied Physics Letters, 89.

Haase, K. and Pelling, A. E. (2015). Investigating cell mechanics with atomic force microscopy. Journal of The Royal Society Interface, 12(104):20140970-20140970. 
Holzapfel, G. A. (2000). Nonlinear solid mechanics: A continuum approach for engineering. John Wiley \& Sons.

Honda, H., Kondo, T., Zhao, Q.-L., Feril, L. B., and Kitagawa, H. (2004). Role of intracellular calcium ions and reactive oxygen species in apoptosis induced by ultrasound. Ultrasound in medicine \& biology, 30(5):683-92.

Huang, S., Chen, Z., Pugno, N., Chen, Q., and Wang, W. (2014). A novel model for porous scaffold to match the mechanical anisotropy and the hierarchical structure of bone. Materials Letters, $122: 315-319$.

Ilinskii, Y. A., Meegan, G. D., Zabolotskaya, E. A., and Emelianov, S. Y. (2005). Gas bubble and solid sphere motion in elastic media in response to acoustic radiation force. The Journal of the Acoustical Society of America, 117(4 Pt 1):2338-2346.

Johns, L. D. (2002). Nonthermal effects of therapeutic ultrasound: The frequency resonance hypothesis. Journal of Athletic Training, 37(3):293-299.

Jonieztz, E. (2012). The forces of cancer. Science, 491:S56-S57.

Ketene, A. N., Schmelz, E. M., Roberts, P. C., and Agah, M. (2012). The effects of cancer progression on the viscoelasticity of ovarian cell cytoskeleton structures. Nanomedicine: Nanotechnology, Biology, and Medicine, 8(1):93-102.

Kittel, C. and Krocmer, H. (1980). Thermal Physics. W. H. Freeman, San Francisco, 2nd editio edition.

Koeller, R. C. (1984). Applications of Fractional Calculus to the Theory of Viscoelasticity. Journal of Applied Mechanics, 51(2):299.

Landau, L. D. and Lifshitz, E. M. (1987). Fluid Mechanics, volume 6 of Course of Theoretical Physics. Pergamon Press.

Lejbkowicz, F. and Salzberg, S. (1997). Distinct sensitivity of normal and malignant cells to ultrasound in vitro. Environmental Health Perspectives, 105(SUPPL. 6):1575-1578.

Lejbkowicz, F., Zwiran, M., and Salzberg, S. (1993). The response of normal and malignant cells to ultrasound in vitro. Ultrasound in medicine \& biology, 19(1):75-82. 
Lekka, M., Gil, D., Pogoda, K., Dulińska-Litewka, J., Jach, R., Gostek, J., Klymenko, O., PrauznerBechcicki, S., Stachura, Z., Wiltowska-Zuber, J., Okoń, K., and Laidler, P. (2012a). Cancer cell detection in tissue sections using AFM. Archives of Biochemistry and Biophysics, 518(2):151-156.

Lekka, M., Laidler, P., Gil, D., Lekki, J., Stachura, Z., and Hrynkiewicz, A. Z. (1999). Elasticity of normal and cancerous human bladder cells studied by scanning force microscopy. European Biophysics Journal, 28(4):312-316.

Lekka, M., Pogoda, K., Gostek, J., Klymenko, O., Prauzner-Bechcicki, S., Wiltowska-Zuber, J., Jaczewska, J., Lekki, J., and Stachura, Z. (2012b). Cancer cell recognition - Mechanical phenotype. Micron, 43(12):1259-1266.

Li, Q. S., Lee, G. Y. H., Ong, C. N., and Lim, C. T. (2008). AFM indentation study of breast cancer cells. Biochemical and Biophysical Research Communications, 374(4):609-613.

Lide, D. R., Data, S. R., Board, E. A., Baysinger, G., Chemistry, S., Library, C. E., Berger, L. I., Goldberg, R. N., Division, B., Kehiaian, H. V., Kuchitsu, K., Rosenblatt, G., Roth, D. L., and Zwillinger, D. (2008). CRC Handbook of Chemistry and Physics. CRC handbook of chemistry and physics, Boca Raton, FL, 88th (inte edition.

Mainardi, F. (2012). An historical perspective on fractional calculus in linear viscoelasticity. Fractional Calculus and Applied Analysis, 15(4):712-717.

Maxey, M. R. and Riley, J. J. (1983). Equation of motion for a small rigid sphere in a nonuniform flow. Physics of Fluids, 26(4):883.

Metzler, R. and Klafter, J. (2000). The random walk's guide to anomalous diffusion: a fractional dynamics approach. Physics Reports, 339(1):1-77.

Michelet-Habchi, C., Incerti, S., Aguer, P., Barberet, P., Gontier, E., Guinefolleau, T., Moretto, P., Pouthier, A., Pouthier, T., and Smith, R. W. (2005). 3D imaging of microscopic structures using a proton beam. IEEE Transactions on Nuclear Science, 52(3):612-617.

Mizrahi, N., Zhou, E. H., Lenormand, G., Krishnan, R., Weihs, D., Butler, J. P., Weitz, D. a., Fredberg, J. J., and Kimmel, E. (2012). Low intensity ultrasound perturbs cytoskeleton dynamics. Soft Matter, $8: 2438$. 
Nikkhah, M., Strobl, J. S., De Vita, R., and Agah, M. (2010). The cytoskeletal organization of breast carcinoma and fibroblast cells inside three dimensional (3-D) isotropic silicon microstructures. Biomaterials, 31(16):4552-4561.

Nutting, P. (1921). A new general law of deformation. Journal of the Franklin Institute, 191(5):679685.

Ohshima, Y. N. and Nishio, I. (2001). Colloidal crystal: Bead-spring lattice immersed in viscous media. Journal of Chemical Physics, 114(19):8649-8658.

Or, M. and Kimmel, E. (2009). Modeling Linear Vibration of Cell Nucleus in Low Intensity Ultrasound Field. Ultrasound in Medicine and Biology, 35(6):1015-1025.

Paszek, M. J., DuFort, C. C., Rossier, O., Bainer, R., Mouw, J. K., Godula, K., Hudak, J. E., Lakins, J. N., Wijekoon, A. C., Cassereau, L., Rubashkin, M. G., Magbanua, M. J., Thorn, K. S., Davidson, M. W., Rugo, H. S., Park, J. W., Hammer, D. a., Giannone, G., Bertozzi, C. R., and Weaver, V. M. (2014). The cancer glycocalyx mechanically primes integrin-mediated growth and survival. Nature, 511(7509):319-25.

Prabhune, M., Belge, G., Dotzauer, A., Bullerdiek, J., and Radmacher, M. (2012). Comparison of mechanical properties of normal and malignant thyroid cells. Micron, 43(12):1267-1272.

Pugno, N. M., Bosia, F., and Abdalrahman, T. (2012). Hierarchical fiber bundle model to investigate the complex architectures of biological materials. Physical Review E - Statistical, Nonlinear, and Soft Matter Physics, 85.

Rebelo, L. M., de Sousa, J. S., Mendes Filho, J., and Radmacher, M. (2013). Comparison of the viscoelastic properties of cells from different kidney cancer phenotypes measured with atomic force microscopy. Nanotechnology, 24(5):055102.

Rodriguez, M. L., McGarry, P. J., and Sniadecki, N. J. (2013). Review on Cell Mechanics: Experimental and Modeling Approaches. Applied Mechanics Reviews, 65(6):060801.

Schiessel, H. and Blumen, A. (1993). Hierarchical analogues to fractional relaxation equations. Journal of Physics A: Mathematical and General, 26(19):5057-5069. 
Schuster, A., Schwab, T., Bischof, M., Klotz, M., Lemor, R., Degel, C., and Schäfer, K. H. (2013). Cell specific ultrasound effects are dose and frequency dependent. Annals of Anatomy, 195(1):5767.

Tschoegl, N. (1989). The phenomenological theory of linear viscoelastic behavior: an introduction. Springer New York.

Wolfram, S. (2003). The Mathematica Book. Wolfram Media, Inc.

\section{List of Tables with enclosed captions}

Table 1: Synoptic table with models and adopted parameters

\begin{tabular}{c|cccccc}
\hline & \multicolumn{5}{c}{ Parameters } \\
\cline { 2 - 7 } Models & $\boldsymbol{\alpha}_{\mathbf{1}}$ & $\boldsymbol{C}_{\boldsymbol{\alpha}_{\mathbf{1}}}$ & $\boldsymbol{\alpha}_{\boldsymbol{2}}$ & $\boldsymbol{C}_{\boldsymbol{\alpha}_{\mathbf{2}}}$ & $\boldsymbol{\alpha}_{\mathbf{3}}$ & $\boldsymbol{C}_{\boldsymbol{\alpha}_{\mathbf{3}}}$ \\
\hline Elastic & 0 & $\rightarrow \infty$ & - & - & 0 & $c_{0 G}$ \\
Viscous & 0 & $\rightarrow \infty$ & - & - & 1 & $c_{1 \mu}$ \\
$\mathbf{V}$ & 0 & $c_{0 G}$ & 1 & $c_{1 \mu}$ & 0 & $\rightarrow \infty$ \\
$\mathbf{M}$ & 0 & $\rightarrow 0$ & 1 & $c_{1 \mu}$ & 0 & $c_{0 G}$ \\
SLK & 0 & $c_{0 G}$ & 1 & $c_{1 \mu}$ & 0 & $c_{0 G}$ \\
SLK_1 & 0.5 & $C_{0.5}$ & 1 & $c_{1 \mu}$ & 0 & $c_{0 G}$ \\
SLK_2 & 0 & $c_{0 G}$ & 0.5 & $C_{0.5}$ & 0 & $c_{0 G}$ \\
SLK_3 & 0 & $c_{0 G}$ & 1 & $c_{1 \mu}$ & 0.5 & $C_{0.5}$ \\
\hline
\end{tabular}




\section{List of Figures with enclosed captions}

a)

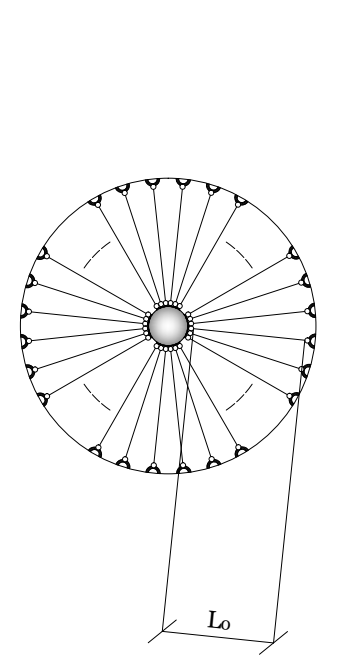

b)

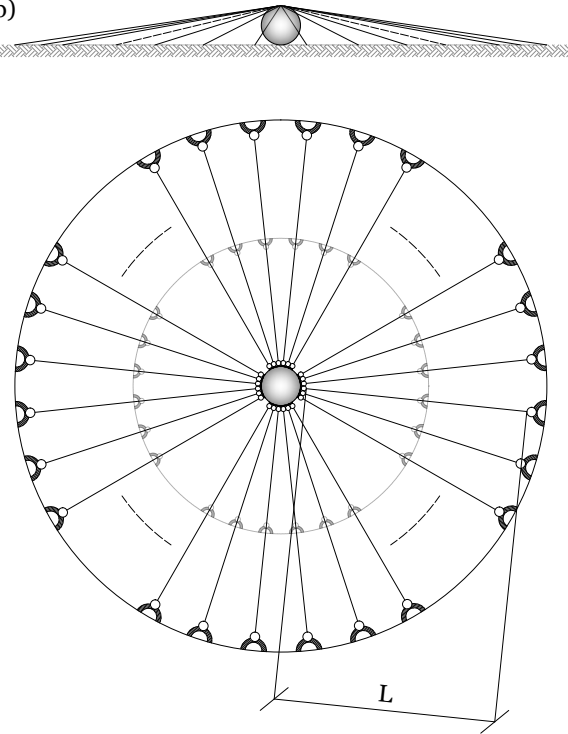

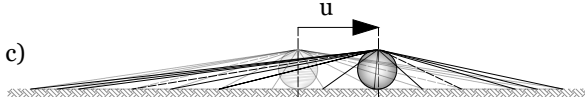

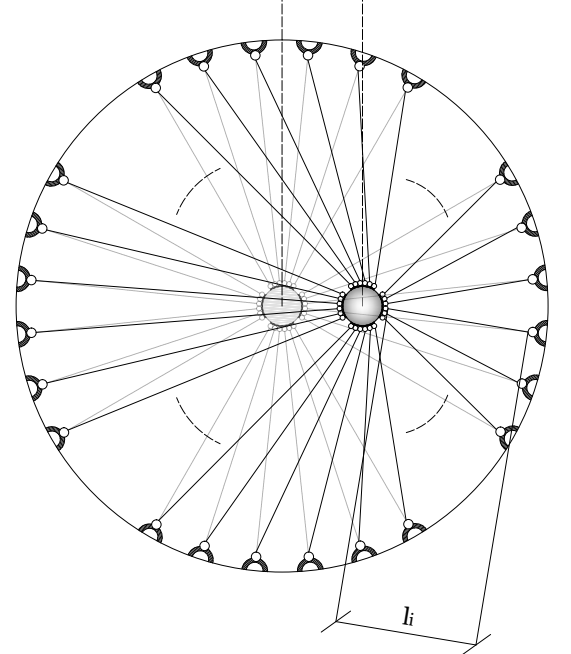

Figure 1: Cartoon -with plan and lateral views- of the elemental cell cytoskeleton structure: a) initial (stress-free) unknown configuration; b) adherent cell with non-linearly prestretched/prestressed filaments (reference configuration); c) small-on-large cell deformation induced by nucleus displacement (current configuration) 


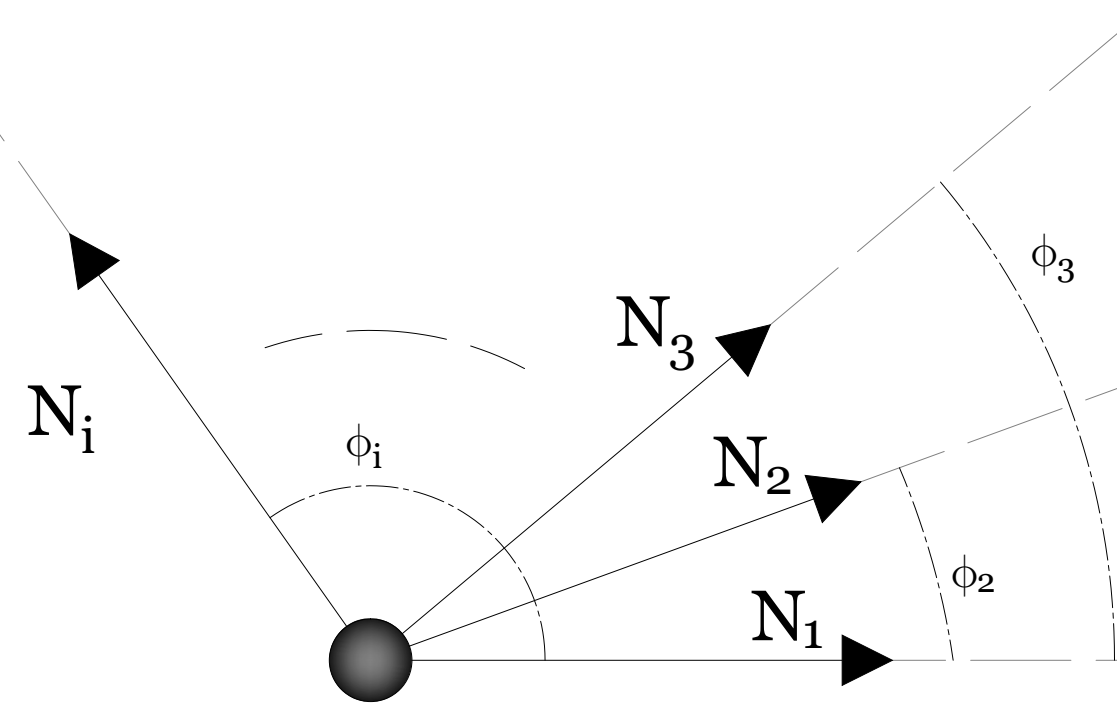

Figure 2: Illustration of how the axial forces kindled in each string contribute to the equilibrium of the nucleus: the angles $\phi_{j}$ are referred to the actual (displaced) nucleus position. 


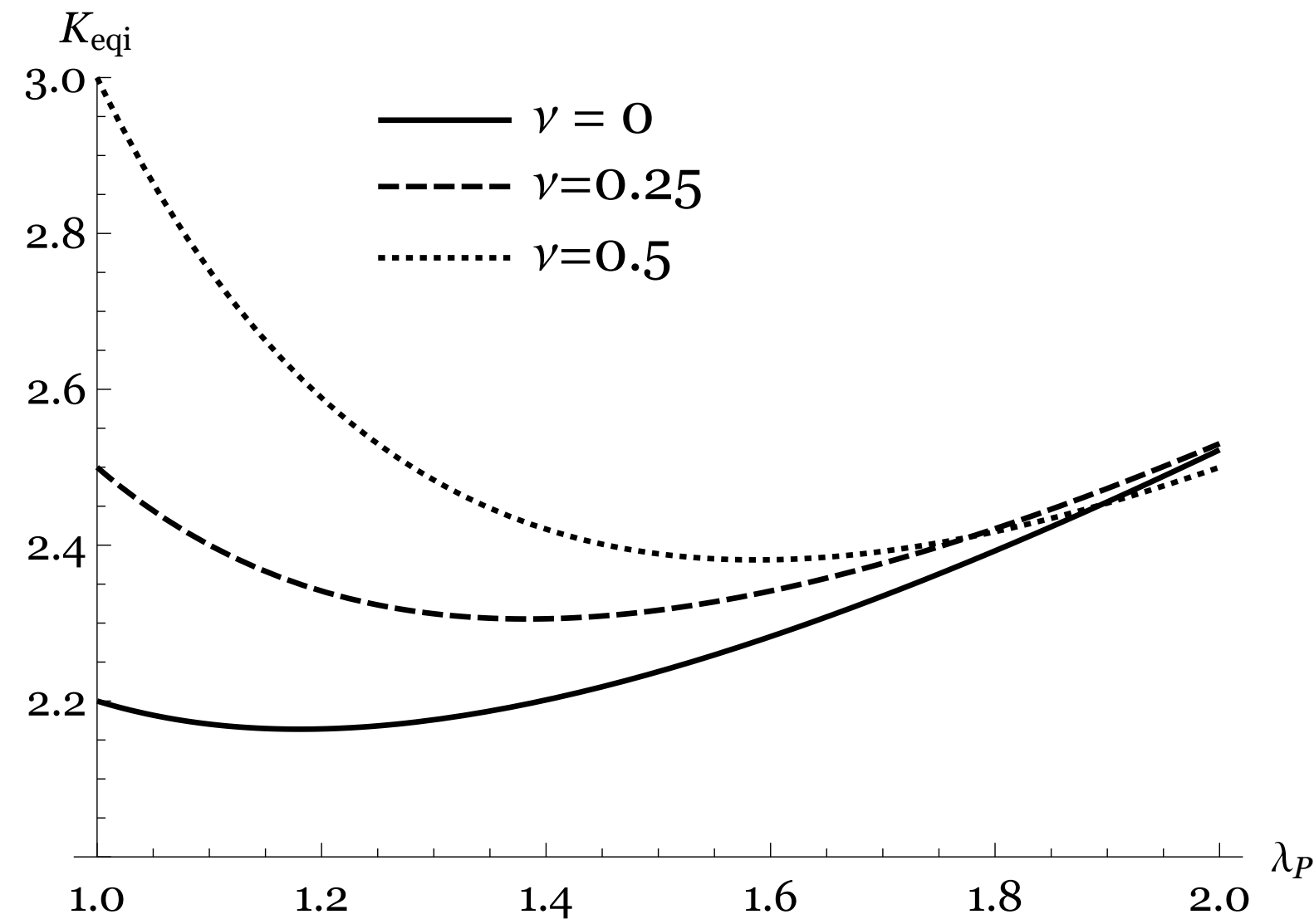

Figure 3: Plot of the dimensionless contribution of a filament to the cell stiffness as function of the prestretch: note that -for different Poisson's ratio values- a minimum is always highlighted. 

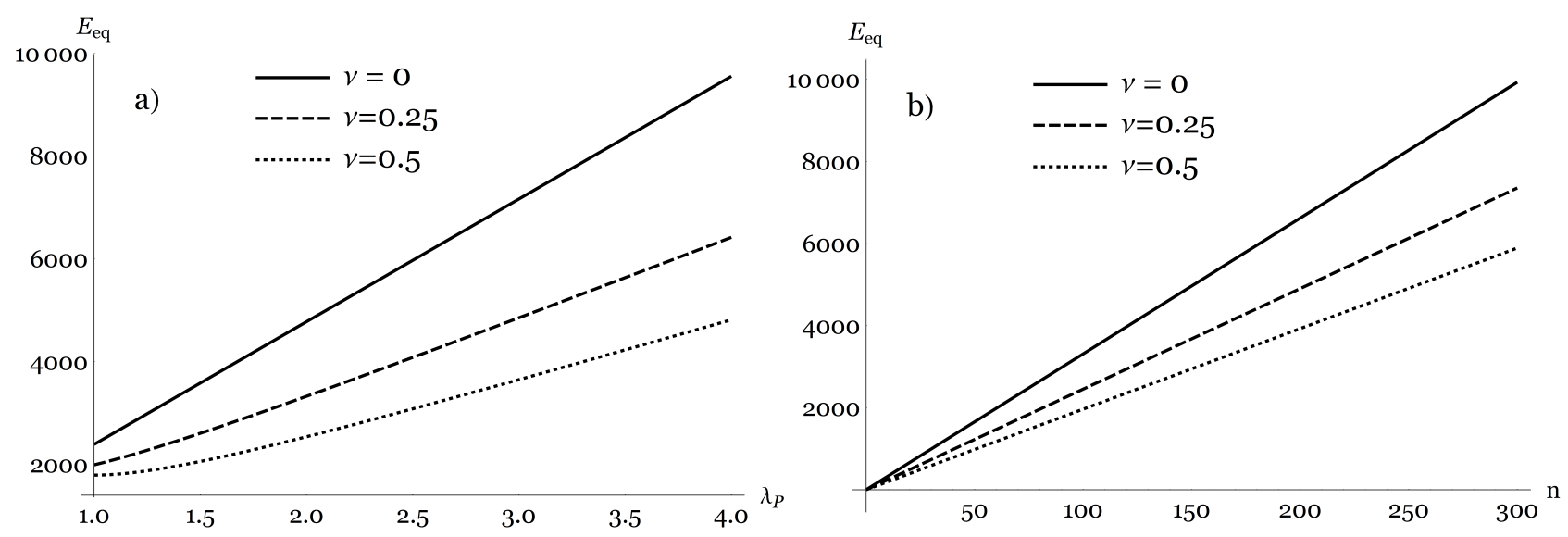

Figure 4: Parametric analyses for the equivalent Young modulus of a single-cell system for different Poisson's ratios: a) elastic stiffness versus prestretch with fixed number of active filaments (75); b) elastic stiffness versus number of (active) filaments, with prescribed prestretch value $\left(\lambda_{p}=1.3\right)$. 


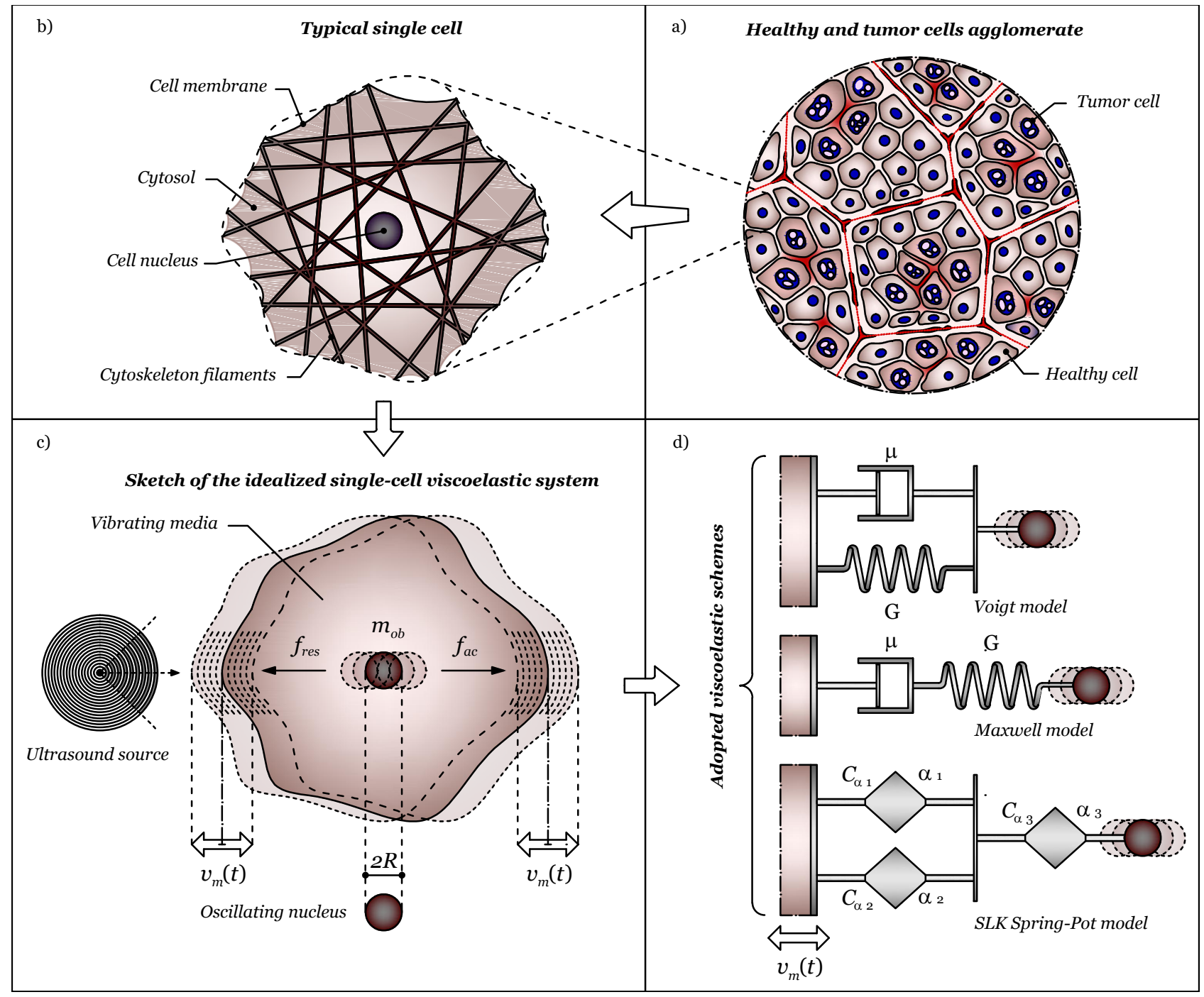

Figure 5: Cartoon of the idealized single-cell system: $(a)$ healthy and tumor cells agglomerate; $(b)$ typical cell unit, with nucleus and cytoskeleton structure embedded in the cytosol and confined by the lipid bilayer cell membrane; $(c)$ idealized single-cell system with cell nucleus oscillating in a viscoelastic environment under the action of radiating ultrasound source; $(d)$ adopted viscoelastic schemes (Voigt, Maxwell and generalized Spring-Pot based Standard Linear Kelvin models). 

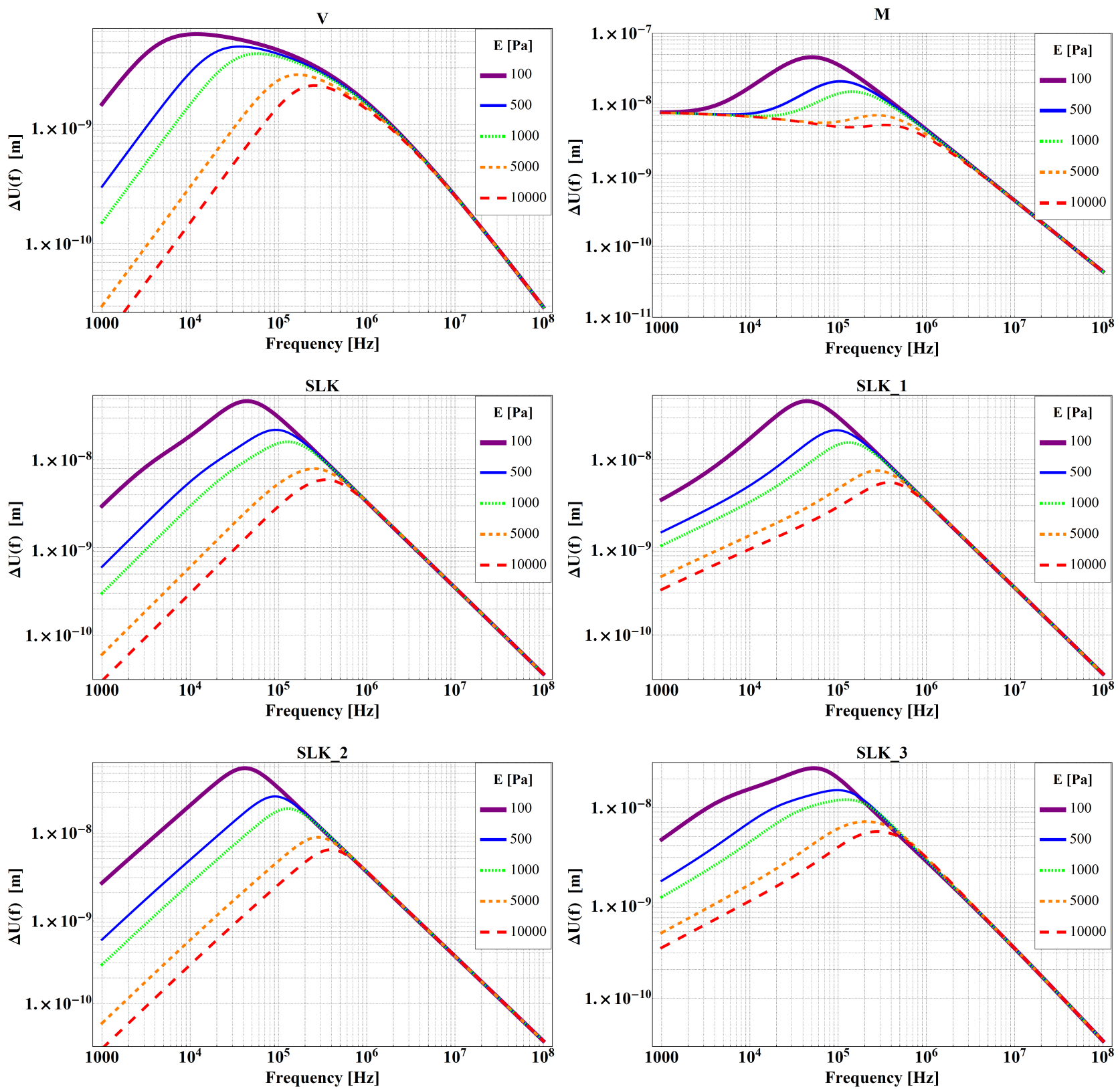

Figure 6: Sensitivity analysis for the frequency response of the cyclic displacement amplitude of a spherical object $(R=1 \mu \mathrm{m})$ with respect to its surroundings with prescribed viscosity $(\mu=$ $\left.10^{-3} \mathrm{~Pa} \times s\right)$ and varying Young modulus $(E=100,500,1000,5000,10000 \mathrm{~Pa}):(\mathbf{V})$ Voigt; $(\mathbf{M})$ Maxwell; (SLK) Standard Linear Kelvin; (SLK_1) generalized Standard Linear Kelvin with springpot in position 1, (SLK_2) 2 and (SLK_3) 3, with $\alpha=0.5$. 

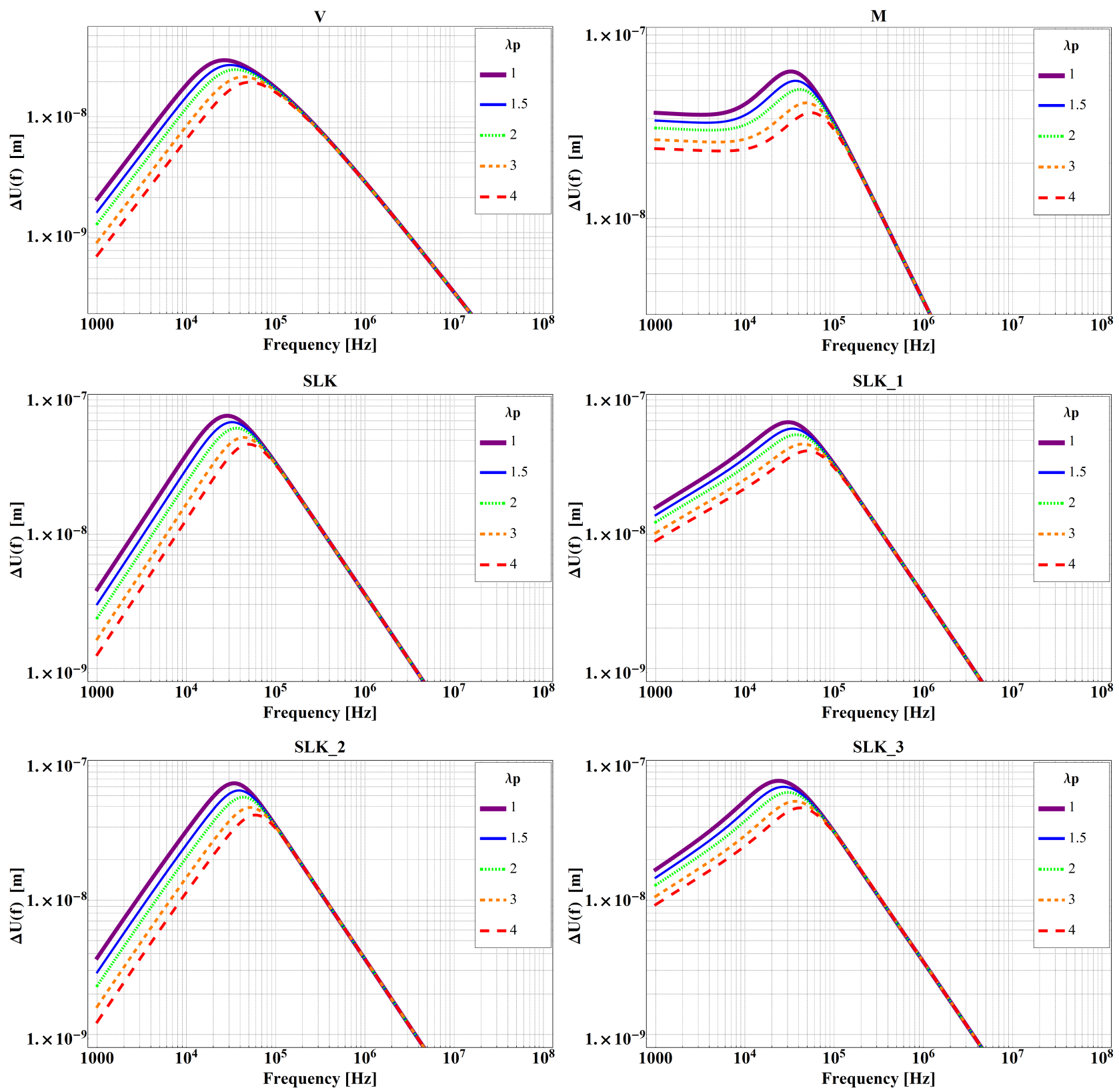

Figure 7: Sensitivity analysis for the frequency response of the cyclic displacement amplitude of a spherical object $(R=1 \mu \mathrm{m})$ with respect to its surroundings with prescribed viscosity $(\mu=$ $\left.10^{-3} \mathrm{~Pa} \times s\right)$, tangent Young modulus $\left(E=2.6 \times 10^{9} \mathrm{~Pa}\right)$ and Poisson ratio $(\nu=0.4)$ of the microfilaments, for a fixed number of active filaments $(n=50)$, varying the level of prestretch $\left(\lambda_{p}=1,1.5,2,3,4\right):(\mathbf{V})$ Voigt; (M) Maxwell; (SLK) Standard Linear Kelvin; (SLK_1) generalized Standard Linear Kelvin with spring-pot in position 1, (SLK_2) 2 and (SLK_3) 3, with $\alpha=0.5$. 

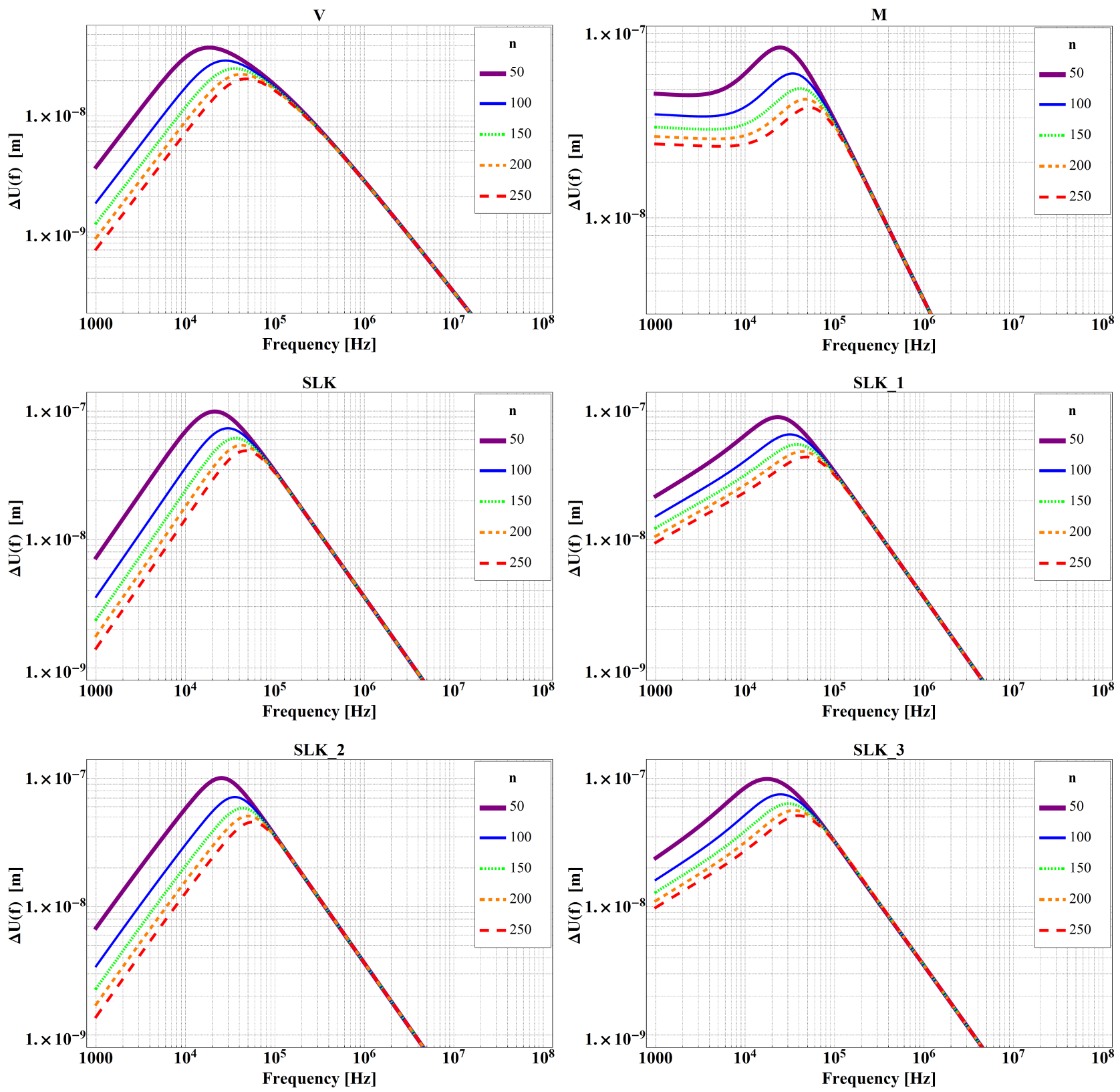

Figure 8: Sensitivity analysis for the frequency response of the cyclic displacement amplitude of a spherical object $(R=1 \mu \mathrm{m})$ with respect to its surroundings with prescribed viscosity $(\mu=$ $\left.10^{-3} \mathrm{~Pa} \times s\right)$, tangent Young modulus $\left(E=2.6 \times 10^{9} \mathrm{~Pa}\right)$ and Poisson ratio $(\nu=0.4)$ of the microfilaments, for a fixed level of prestretch $\left(\lambda_{p}=1.3\right)$, varying number of active microfilaments ( $n=50$, 100, 150, 200, 250): (V) Voigt; (M) Maxwell; (SLK) Standard Linear Kelvin; (SLK_1) generalized Standard Linear Kelvin with spring-pot in position 1, (SLK_2) 2 and (SLK_3) 3, with $\alpha=0.5$. 